\title{
An analytic approach to a class of fractional differential-difference equations of rational type via symbolic computation
}

\author{
Ismail Aslan*
}

\section{Communicated by S. G. Georgiev}

Fractional derivatives are powerful tools in solving the problems of science and engineering. In this paper, an analytical algorithm for solving fractional differential-difference equations in the sense of Jumarie's modified Riemann-Liouville derivative has been described and demonstrated. The algorithm has been tested against time-fractional differentialdifference equations of rational type via symbolic computation. Three examples are given to elucidate the solution procedure. Our analyses lead to closed form exact solutions in terms of hyperbolic, trigonometric, and rational functions, which might be subject to some adequate physical interpretations in the future. Copyright $\odot 2013$ John Wiley \& Sons, Ltd.

Keywords: fractional derivative; differential-difference equation; (G'/G)-expansion method

\section{Introduction}

Fractional calculus, which deals with the derivatives and integrals of fractional (real or complex) order, has found its applications in various areas of science and engineering. To make mention, a few areas of science and engineering are as follows: bioengineering, robotics, electrochemical processes, viscoelasticity theory, electro analytical chemistry, noise simulations, transport processes, electrical circuits, electromagnetic theory, porous media, polarography theory, fractal theory, colored noise, dielectric polarization, viscoelastic materials, and so on. To get a feel about these applications, we refer the interested reader to Ref. [1-5]. In the literature, on the basis of the problem under investigation, there exist vast different definitions of fractional derivatives such as Riemann-Liouville derivative, GrünwaldLetnikov derivative, Sonin-Letnikov derivative, Riesz derivative, Caputo derivative, Marchaud derivative, Weyl derivative, and others. Among those, Grünwald-Letnikov derivative, Riemann-Liouville derivative, and Caputo derivative are widely used. Grünwald-Letnikov derivative and Riemann-Liouville derivative are equivalent if the functions they act on are sufficiently smooth, and Riemann-Liouville derivative is meaningful under weaker smoothness requirements. In addition, Caputo fractional derivative first computes an ordinary derivative followed by a fractional integral, whereas Riemann-Liouville fractional derivative is computed in the reverse order. Moreover, a number of local versions of fractional derivatives also presented for the analysis of local behavior of fractional models such as Jumarie's modified Riemann-Liouville derivative [6], Cresson's derivative [7], and Kolwankar-Gangal local derivative [8]. On the other hand, the recent appearance of fractional differential equations (FDEs) as adequate models in science and engineering made it necessary to develop methods of solutions (both analytical and numerical) These methods include finite difference method [9], finite element method [10], differential transform method [11], Adomian decomposition method [12], variational iteration method [13], homotopy perturbation method [14], first integral method [15], fractional sub-equation method [16], B-spline function method [17], Tau method [18], homotopy analysis method [19,20], and collocation method [21]. Although these methods lead to exact solutions in some special cases, exact solutions are much needed in engineering applications. Besides, some of the just-mentioned methods use a transformation in order to reduce equations into simpler forms, whereas others provide a solution in an infinite series form converging to the desired exact solution.

Differential-difference equations (DDEs), also known as lattices or networks, play an important role in many scientific fields from engineering to physics, chemistry, biology, and so on. Applications of DDEs are diverse, for instance, currents in electrical networks, pulses in biological chains, particle vibrations in lattices, chemical reactions, and wave phenomena in fluids. Since the time of Fermi, Pasta, and Ulam [22], quite a few lattices have been discovered, in particular, Volterra lattice, Toda lattice, discrete (modified) KdV equation,

Department of Mathematics, Izmir Institute of Technology, Urla, Izmir 35430, Turkey

*Correspondence to: Ismail Aslan, Department of Mathematics, İmir Institute of Technology, Urla, Izmir 35430, Turkey.

${ }^{\dagger}$ E-mail: ismailaslan@iyte.edu.tr 
Ablowitz-Ladik lattice, and Suris lattice [23]. In addition to their physical relevance, DDEs also play an important role in numerical simulations of nonlinear partial differential equations. Consequently, considerable attention has been given to the solution of DDEs. Many powerful methods for solving such equations appeared in the open literature, such as Exp-function method [24], Casoratian technique [25], homotopy perturbation method [26], ADM-Padé technique [27], Hirota's bilinear method [28], (G'/G)-expansion method [29], and Tau method [30]. Of course, each of the just-stated methods for DDEs has its own advantages and its own defects, and as a result, alternatives may be useful in some instances, for getting different insights into the problem. As far as we could verify, less work is being performed for the symbolic computation of exact solutions to DDEs in the form $\dot{u}_{n}=R\left(u_{n-1}, u_{n}, u_{n+1}\right)$, where $R$ is a rational function of its arguments, whereas there has been a considerable amount of work performed in finding exact solutions to DDEs in the form $\dot{u}_{n}=P\left(u_{n-1}, u_{n}, u_{n+1}\right)$, where $P$ is a polynomial function of its arguments. Here, we should note that we deal with a DDE in which the spatial variable $n$ is discrete, whereas the time $t$ is kept continuous. This means that there will be a different problem for each $n$. Because of their structure, DDEs can be considered as hybrid systems as well. To broaden the class of DDEs and study various processes described by them is of interest. In a previous review paper, He et al. [31] suggested for the first time that DDEs can be extended in the context of fractional calculus, namely, the reformulation of DDEs with fractional order. In fact, it is logical to do research in this direction. Thus, the main object of our study will be the investigation of time-fractional DDEs in the form

$$
D_{t}^{\alpha} u_{n}=R\left(u_{n-1}, u_{n}, u_{n+1}\right), 0<\alpha \leq 1
$$

where $D_{t}^{\alpha}$ denotes Jumarie's modified Riemann-Liouville derivative (in time) of order $\alpha$. Here, the dependent variable $u_{n}$ is assumed to be a function $u(n, t)$ of a lattice variable $n \in \square$. We call Equation (1) as a rational type in the sense that the right-hand side is a rational function of dependent variables. At the same time, the authors [31] have also pointed out new directions in nonlinear science by proposing three standard variational iteration algorithms for solving differential equations, integro-differential equations, FDEs, fractal differential equations, DDEs, and fractional/fractal DDEs.

We shall show here how the so-called (G'/G)-expansion method [32], which is used for solving nonlinear evolution equations as well as FDEs [33,34], can be extended to time-fractional DDEs. In comparison with other analytical schemes, the main advantage of (G'/G)expansion method is in its flexibility and ability to solve nonlinear evolution equations accurately and conveniently. Being computer oriented, it reduces the problems of those solving a system of algebraic equations.

This paper is organized as follows. In the next section, a short background on the definition of the Jumarie's modified RiemannLiouville derivative is given. In Section 3, we shall describe the method itself. In Section 4, three time-fractional DDEs of rational type are analyzed. Finally, Section 5 is devoted to concluding remarks.

\section{Preliminaries}

The Jumarie's modified Riemann-Liouville derivative of order $\alpha$ is defined as

$$
\begin{gathered}
D_{t}^{\alpha} f(t)=\frac{1}{\Gamma(-\alpha)} \int_{0}^{t}(t-\xi)^{-\alpha-1}(f(\xi)-f(0)) d \xi, \alpha<0, \\
D_{t}^{\alpha} f(t)=\frac{1}{\Gamma(1-\alpha)} \frac{d}{d t} \int_{0}^{t}(t-\xi)^{-\alpha}(f(\xi)-f(0)) d \xi, 0<\alpha<1, \\
D_{t}^{\alpha} f(t)=\left(f^{(\alpha-n)}(t)\right)^{(n)}, n \leq \alpha<n+1, n \geq 1 .
\end{gathered}
$$

This fractional derivative was successfully implemented to fractional Laplace problems [35], fractional variational calculus [36], and probability calculus [37]. Jumarie's modified Riemann-Liouville derivative has many interesting properties: the $\alpha$ order derivative of a constant is zero and it can be applied to both differentiable and nondifferentiable functions. Some formulas and results can be found in [6]. For example,

$$
\begin{gathered}
D_{t}^{\alpha} t^{\gamma}=\frac{\Gamma(1+\gamma)}{\Gamma(1+\gamma-\alpha)} t^{\gamma-\alpha}, \gamma>0, \\
D_{t}^{\alpha}(f(t) g(t))=g(t) D_{t}^{\alpha} f(t)+f(t) D_{t}^{\alpha} g(t), \\
D_{t}^{\alpha} f(g(t))=f_{g}^{\prime}(g(t)) D_{t}^{\alpha} g(t)=D_{g}^{\alpha} f(g(t))\left(g_{t}^{\prime}\right)^{\alpha},
\end{gathered}
$$

which are direct consequences of the equality $d^{\alpha} x(t)=\Gamma(1+\alpha) d x(t)$. 


\section{Methodology}

In a recent study, $\mathrm{Li}$ and $\mathrm{He}$ [38] proved that FDEs in the sense of Jumarie's modified Riemann-Liouville derivative can be easily turned into ODEs so that anyone can deal with fractional calculus with ease. The authors proposed a fractional complex transformation in the form

$$
\xi=\frac{p x^{\alpha}}{\Gamma(1+\alpha)}+\frac{q y^{\beta}}{\Gamma(1+\beta)}+\frac{r t^{\gamma}}{\Gamma(1+\gamma)}, 0<\alpha \leq 1,0<\beta \leq 1,0<\gamma \leq 1,
$$

which is a variant of the wave transformation $\eta=k x+l y+m t$, where $k, l$, and $m$ are constants to be determined. Now, to illustrate the basic idea of the method, we consider a general system of $M$ fractional DDEs of rational type

$$
R\left(\mathbf{u}_{\mathbf{n}+\mathbf{p}_{1}}(\mathbf{x}), \ldots, \mathbf{u}_{\mathbf{n}+\mathbf{p}_{k}}(\mathbf{x}), \ldots, \mathbf{u}_{\mathbf{n}+\mathbf{p}_{1}}^{\alpha}(\mathbf{x}), \ldots, \mathbf{u}_{\mathbf{n}+\mathbf{p}_{k}}^{\alpha}(\mathbf{x}), \ldots, \mathbf{u}_{\mathbf{n}+\mathbf{p}_{1}}^{(r \alpha)}(\mathbf{x}), \ldots, \mathbf{u}_{\mathbf{n}+\mathbf{p}_{k}}^{(r \alpha)}(\mathbf{x})\right)=0,0<\alpha \leq 1,
$$

where the dependent variable $\mathbf{u}_{\mathbf{n}}$ have $M$ components $u_{i, \mathbf{n}}$ and so do its shifts; the continuous variable $\mathbf{x}$ has $N$ components $x_{i}$; the discrete variable $\mathbf{n}$ has $Q$ components $n_{j}$; the $k$ shift vectors $\mathbf{p}_{i} \in \square^{Q}$; and $\mathbf{u}^{(r \alpha)}(\mathbf{x})$ denotes the collection of Jumarie's modified Riemann-Liouville derivative terms of order $r \alpha$. As mentioned earlier, using the fractional complex transformation

$$
\mathbf{u}_{\mathbf{n}+\mathbf{p}_{s}}(\mathbf{x})=\mathbf{U}_{\mathbf{n}+\mathbf{p}_{s}}\left(\xi_{\mathbf{n}}\right), \xi_{\mathbf{n}}=\sum_{i=1}^{Q} d_{i} n_{i}+\sum_{j=1}^{N} \frac{c_{j}}{\Gamma(1+\alpha)} x_{j}^{\alpha}+\zeta_{,}(s=1,2, \ldots, k),
$$

where the coefficients $c_{1}, c_{2}, \ldots, c_{N}, d_{1}, d_{2}, \ldots, d_{Q}$ and the phase $\zeta$ are all constants and the symbol $\Gamma$ is the gamma function, we can rewrite Equation (9) as a system of DDEs of integer order in the form

$$
R\left(\mathbf{U}_{\mathbf{n}+\mathbf{p}_{1}}\left(\xi_{\mathbf{n}}\right), \ldots, \mathbf{U}_{\mathbf{n}+\mathbf{p}_{k}}\left(\xi_{\mathbf{n}}\right), \ldots, \mathbf{U}_{\mathbf{n}+\mathbf{p}_{1}}^{\prime}\left(\xi_{\mathbf{n}}\right), \ldots, \mathbf{U}_{\mathbf{n}+\mathbf{p}_{k}}^{\prime}\left(\xi_{\mathbf{n}}\right), \ldots, \mathbf{U}_{\mathbf{n}+\mathbf{p}_{1}}^{(r)}\left(\xi_{\mathbf{n}}\right), \ldots, \mathbf{U}_{\mathbf{n}+\mathbf{p}_{k}}^{(r)}\left(\xi_{\mathbf{n}}\right)\right)=0
$$

According to the basic ( $\left.G^{\prime} / G\right)$-expansion method, we assume that the solution(s) of Equation (11) can be expressed in the form

$$
\mathbf{U}_{\mathbf{n}}\left(\xi_{\mathbf{n}}\right)=\sum_{l=0}^{m} a_{l}\left(\frac{G^{\prime}\left(\xi_{\mathbf{n}}\right)}{G\left(\xi_{\mathbf{n}}\right)}\right)^{l}, a_{m} \neq 0,
$$

where $m$ is a positive integer, $a_{i}$ 's are constants to be determined, and $G\left(\xi_{\mathbf{n}}\right)$ is a solution of the second-order linear ordinary differential equation

$$
G^{\prime \prime}\left(\xi_{\mathbf{n}}\right)+\lambda G^{\prime}\left(\xi_{\mathbf{n}}\right)+\mu G\left(\xi_{\mathbf{n}}\right)=0,
$$

where $\lambda$ and $\mu$ are arbitrary parameters and prime denotes derivative with respect to $\xi_{\mathbf{n}}$. The general solution of Equation (13) is well known to us. Thus, three cases follow:

$$
\begin{gathered}
\frac{G^{\prime}\left(\xi_{\mathbf{n}}\right)}{G\left(\xi_{\mathbf{n}}\right)}=-\frac{\lambda}{2}+\frac{\sqrt{\lambda^{2}-4 \mu}}{2}\left(\frac{C_{1} \cosh \left(\left(\sqrt{\lambda^{2}-4 \mu} / 2\right) \xi_{\mathbf{n}}\right)+C_{2} \sinh \left(\left(\sqrt{\lambda^{2}-4 \mu} / 2\right) \xi_{\mathbf{n}}\right)}{C_{1} \sinh \left(\left(\sqrt{\lambda^{2}-4 \mu} / 2\right) \xi_{\mathbf{n}}\right)+C_{2} \cosh \left(\left(\sqrt{\lambda^{2}-4 \mu} / 2\right) \xi_{\mathbf{n}}\right)}\right), \lambda^{2}-4 \mu>0, \\
\frac{G^{\prime}\left(\xi_{\mathbf{n}}\right)}{G\left(\xi_{\mathbf{n}}\right)}=-\frac{\lambda}{2}+\frac{\sqrt{4 \mu-\lambda^{2}}}{2}\left(\frac{-C_{1} \sin \left(\left(\sqrt{4 \mu-\lambda^{2}} / 2\right) \xi_{\mathbf{n}}\right)+C_{2} \cos \left(\left(\sqrt{4 \mu-\lambda^{2}} / 2\right) \xi_{\mathbf{n}}\right)}{C_{1} \cos \left(\left(\sqrt{4 \mu-\lambda^{2}} / 2\right) \xi_{\mathbf{n}}\right)+C_{2} \sin \left(\left(\sqrt{4 \mu-\lambda^{2}} / 2\right) \xi_{\mathbf{n}}\right)}\right), \lambda^{2}-4 \mu<0, \\
\frac{G^{\prime}\left(\xi_{\mathbf{n}}\right)}{G\left(\xi_{\mathbf{n}}\right)}=-\frac{\lambda}{2}+\frac{C_{1}}{C_{1} \xi_{\mathbf{n}}+C_{2}}, \lambda^{2}-4 \mu=0,
\end{gathered}
$$

where $C_{1}$ and $C_{2}$ are arbitrary constants. A straightforward calculation leads to the identity

$$
\xi_{\mathbf{n}+\mathbf{p}_{s}}=\xi_{\mathbf{n}}+\varphi_{s}, \quad \varphi_{s}=p_{s 1} d_{1}+p_{s 2} d_{2}+\cdots+p_{s Q} d_{Q}
$$

where $p_{s j}$ is the $j$ th component of the shift vector $\mathbf{p}_{s}$. Hence, considering trigonometric/hyperbolic function identities and using the expressions (14)-(16) as well as (17), we derive

$$
\frac{G^{\prime}\left(\xi_{\mathbf{n} \pm \mathbf{p}_{s}}\right)}{G\left(\xi_{\mathbf{n} \pm \mathbf{p}_{s}}\right)}=-\frac{\lambda}{2}+\frac{\frac{\lambda}{2}+\frac{G^{\prime}\left(\xi_{\mathbf{n}}\right)}{G\left(\xi_{\mathbf{n}}\right)} \pm \varepsilon \frac{\sqrt{\delta+\varepsilon\left(\lambda^{2}-4 \mu\right)}}{2} f\left(\frac{\sqrt{\delta+\varepsilon\left(\lambda^{2}-4 \mu\right)}}{2} \varphi_{s}\right)}{1 \pm \frac{2}{\sqrt{\delta+\varepsilon\left(\lambda^{2}-4 \mu\right)}}\left(\frac{G^{\prime}\left(\xi_{\mathbf{n}}\right)}{G\left(\xi_{\mathbf{n}}\right)}+\frac{\lambda}{2}\right) f\left(\frac{\sqrt{\delta+\varepsilon\left(\lambda^{2}-4 \mu\right)}}{2} \varphi_{s}\right)}
$$


where $\varepsilon \in\{0, \pm 1\}$ and $\delta \in\{0,4\}$, whereas

$$
\begin{gathered}
f\left(\left(\sqrt{\delta+\varepsilon\left(\lambda^{2}-4 \mu\right)} / 2\right) \varphi_{s}\right)=\tanh \left(\left(\sqrt{\lambda^{2}-4 \mu} / 2\right) \varphi_{s}\right), \varepsilon=1, \delta=0, \lambda^{2}-4 \mu>0, \\
f\left(\left(\sqrt{\delta+\varepsilon\left(\lambda^{2}-4 \mu\right)} / 2\right) \varphi_{s}\right)=\tan \left(\left(\sqrt{4 \mu-\lambda^{2}} / 2\right) \varphi_{s}\right), \varepsilon=-1, \delta=0, \lambda^{2}-4 \mu<0, \\
f\left(\left(\sqrt{\delta+\varepsilon\left(\lambda^{2}-4 \mu\right)} / 2\right) \varphi_{s}\right)=\varphi_{s}, \varepsilon=0, \delta=4, \lambda^{2}-4 \mu=0 .
\end{gathered}
$$

We therefore obtain a uniform shift formula in the form

$$
\mathbf{U}_{\mathbf{n}+\mathbf{p}_{s}}\left(\xi_{\mathbf{n}}\right)=\sum_{l=0}^{m} a_{l}\left(-\frac{\lambda}{2}+\frac{\frac{\lambda}{2}+\frac{G^{\prime}\left(\xi_{\mathbf{n}}\right)}{G\left(\xi_{\mathbf{n}}\right)} \pm \varepsilon \frac{\sqrt{\delta+\varepsilon\left(\lambda^{2}-4 \mu\right)}}{2} f\left(\frac{\sqrt{\delta+\varepsilon\left(\lambda^{2}-4 \mu\right)}}{2} \varphi_{s}\right)}{1 \pm \frac{2}{\sqrt{\delta+\varepsilon\left(\lambda^{2}-4 \mu\right)}}\left(\frac{G^{\prime}\left(\xi_{\mathbf{n}}\right)}{G\left(\xi_{\mathbf{n}}\right)}+\frac{\lambda}{2}\right) f\left(\frac{\sqrt{\delta+\varepsilon\left(\lambda^{2}-4 \mu\right)}}{2} \varphi_{s}\right)}\right)^{\prime} .
$$

Balancing the highest order derivative term and the highest order nonlinear term(s) in $\mathbf{U}_{\mathbf{n}}\left(\xi_{\mathbf{n}}\right)$ as in the continuous case, the degree $m$ of Equations (12) and (22) can be easily determined from Equation (11). Because $\mathbf{U}_{\mathbf{n}}+\mathbf{p}_{s}$ can be thought as being of degree zero in $\frac{G^{\prime}\left(\xi_{\mathbf{n}}\right)}{G\left(\xi_{\mathbf{n}}\right)}$, the leading terms of $\mathbf{U}_{\mathbf{n}+\mathbf{p}_{s}}\left(\mathbf{p}_{s} \neq \mathbf{0}\right)$ will not have any effect on the balancing procedure. Substituting Equations (12) and (22) together with (13) into (11), equating the coefficients of $\left(\frac{G^{\prime}\left(\xi_{n}\right)}{G\left(\xi_{n}\right)}\right)^{\prime}(I=0,1,2, \ldots)$ to zero, we obtain a system of nonlinear algebraic equations from which the unspecified constants $a_{i}, d_{i}, c_{j}$, and $k$ can be explicitly found. Finally, substituting these results into Equation (12), one can derive various kind of exact solutions to Equation (9).

Remark 1

The second-order linear equation $G^{\prime \prime}+\lambda G^{\prime}+\mu G=0$ plays a crucial role in the process of the basic ( $\left.G^{\prime} / G\right)$-expansion method, where $\lambda$ and $\mu$ are arbitrary parameters. However, Aslan [39] proved that the parameter $\lambda$ can be set to zero without loss of generality. This idea is more advantageous because it minimizes the number of involved parameters at the beginning as well as leading equivalent results with the original assumption. Hence, from now on, we take $\lambda=0$.

\section{Applications}

We shall point out that we now have at hand the entire framework for extending $\left(G^{\prime} / G\right)$-expansion method to time-fractional DDEs of rational type. In this section, we consider three examples that demonstrate the performance and efficiency of our method.

\section{Example 4.1}

Consider the following time-fractional DDE of rational type:

$$
D_{t}^{\alpha} u_{n}=\frac{u_{n-1}-u_{n+1}}{1+u_{n-1}-u_{n+1}}, 0<\alpha \leq 1
$$

The case $\alpha=1$ of Equation (23), from which the discrete KdV equation can be directly produced, appears in [40]. To discover exact solutions for Equation (23), let us first make the fractional complex transformation

$$
u_{n}=U_{n}\left(\xi_{n}\right), \xi_{n}=d n+\frac{k}{\Gamma(1+\alpha)} t^{\alpha}+\chi
$$

where $d$ and $k$ are real parameters to be specified, whereas $\chi$ denotes the phase shift. On substituting Equation (24) into Equation (23), one obtains

$$
k U_{n}^{\prime}\left(1+U_{n-1}-U_{n+1}\right)-\left(U_{n-1}-U_{n+1}\right)=0,
$$

where prime denotes ordinary derivative with respect to the new independent variable $\xi_{n}$. Then, our procedure suggests looking for a solution of Equation (25) in the form

$$
U_{n}=a_{0}+a_{1}\left(\frac{G^{\prime}}{G}\right), a_{1} \neq 0
$$


4.1.1. Hyperbolic function solutions. In case $\mu<0$, we first derive the shift formulas

$$
U_{n \pm 1}=a_{0}+a_{1}\left(-\frac{\lambda}{2}+\frac{\frac{\lambda}{2}+\frac{G^{\prime}}{G} \pm \frac{\sqrt{\lambda^{2}-4 \mu}}{2} \tanh \left(\frac{\sqrt{\lambda^{2}-4 \mu}}{2} d\right)}{1 \pm \frac{2}{\sqrt{\lambda^{2}-4 \mu}}\left(\frac{\lambda}{2}+\frac{G^{\prime}}{G}\right) \tanh \left(\frac{\sqrt{\lambda^{2}-4 \mu}}{2} d\right)}\right)
$$

in accordance with Equation (22). Substituting Equations (26) and (27) along with (13) into Equation (25), clearing the denominator, and setting the coefficients of $\left(\frac{G^{\prime}}{G}\right)^{l}(I=0,2,4)$ to zero, we derive a system of nonlinear algebraic equations for $a_{0}, a_{1}, d, k$, and $\mu$. Solving the resulting system, we obtain the relation

$$
\lambda=0, a_{0}=a_{0}, a_{1}=\frac{\tanh (d \sqrt{-\mu})}{2 \sqrt{-\mu}}, k=-\frac{\sinh (2 d \sqrt{-\mu})}{\sqrt{-\mu}},
$$

which yields a hyperbolic function solution to Equation (23) as

$$
u_{n}(t)=a_{0}+\frac{1}{2} \tanh (d \sqrt{-\mu})\left(\frac{C_{1} \cosh \left(\sqrt{-\mu} \xi_{n}\right)+C_{2} \sinh \left(\sqrt{-\mu} \xi_{n}\right)}{C_{1} \sinh \left(\sqrt{-\mu} \xi_{n}\right)+C_{2} \cosh \left(\sqrt{-\mu} \xi_{n}\right)}\right),
$$

where $\xi_{n}=d n-\frac{\sinh (2 d \sqrt{-\mu})}{\Gamma(1+\alpha) \sqrt{-\mu}} t^{\alpha}+\chi$, whereas $a_{0}, d, \chi, \mu(<0), C_{1}$, and $C_{2}$ remain arbitrary.

\subsubsection{Trigonometric function solutions. In case $\mu>0$, we first derive the shift formulas}

$$
U_{n \pm 1}=a_{0}+a_{1}\left(-\frac{\lambda}{2}+\frac{\frac{\lambda}{2}+\frac{G^{\prime}}{G} \mp \frac{\sqrt{4 \mu-\lambda^{2}}}{2} \tan \left(\frac{\sqrt{4 \mu-\lambda^{2}}}{2} d\right)}{1 \pm \frac{2}{\sqrt{4 \mu-\lambda^{2}}}\left(\frac{\lambda}{2}+\frac{G^{\prime}}{G}\right) \tan \left(\frac{\sqrt{4 \mu-\lambda^{2}}}{2} d\right)}\right)
$$

in accordance with Equation (22). Substituting Equations (26) and (30) along with (13) into Equation (25), clearing the denominator, and setting the coefficients of $\left(\frac{G^{\prime}}{G}\right)^{l}(I=0,2,4)$ to zero, we derive a system of nonlinear algebraic equations for $a_{0}, a_{1}, d, k$ and $\mu$. Solving the resulting system, we obtain the relation

$$
\lambda=0, a_{0}=a_{0}, a_{1}=\frac{\tan (d \sqrt{\mu})}{2 \sqrt{\mu}}, k=-\frac{\sin (2 d \sqrt{\mu})}{\sqrt{\mu}},
$$

which gives a trigonometric function solution to Equation (23) as

$$
u_{n}(t)=a_{0}+\frac{1}{2} \tan (d \sqrt{\mu})\left(\frac{-C_{1} \sin \left(\sqrt{\mu \xi_{n}}\right)+C_{2} \cos \left(\sqrt{\mu \xi_{n}}\right)}{C_{1} \cos \left(\sqrt{\mu \xi_{n}}\right)+C_{2} \sin \left(\sqrt{\mu \xi_{n}}\right)}\right)
$$

where $\xi_{n}=d n-\frac{\sin (2 d \sqrt{\mu})}{\Gamma(1+\alpha) \sqrt{\mu}} t^{\alpha}+\chi$, whereas $a_{0}, d, \chi_{1} \mu(>0), C_{1}$, and $C_{2}$ remain arbitrary.

4.1.3. Rational function solutions. In case $\mu=0$, we first derive the shift formulas

$$
U_{n \pm 1}=a_{0}+a_{1}\left(-\frac{\lambda}{2}+\frac{\frac{G^{\prime}}{G}+\frac{\lambda}{2}}{1 \pm\left(\frac{G^{\prime}}{G}+\frac{\lambda}{2}\right) d}\right)
$$

in accordance with Equation (22). Substituting Equations (26) and (33) along with (13) into Equation (25), clearing the denominator, and setting the coefficients of $\left(\frac{G^{\prime}}{G}\right)^{\prime}(I=2,4)$ to zero, we derive a system of nonlinear algebraic equations for $a_{0}, a_{1}, d$, and $k$. Solving the resulting system, we obtain the relation

$$
\lambda=0, a_{0}=a_{0}, a_{1}=\frac{d}{2}, k=-2 d,
$$

which yields a rational function solution to Equation (23) as

$$
u_{n}(t)=a_{0}+\frac{d}{2}\left(\frac{C_{1}}{C_{1}\left(d n-\frac{2 d}{\Gamma(1+\alpha)} t^{\alpha}+\chi\right)+C_{2}}\right)
$$

where $a_{0}, d, \chi, C_{1}$, and $C_{2}$ remain arbitrary. 
Remark 2

It is possible to construct some special solutions for Equation (23) by assigning special values to the arbitrary parameters $C_{1}$ and $C_{2}$. For example, if we set ' $C_{1}=0$ and $C_{2} \neq 0$ ' or ' $C_{1} \neq 0$ and $C_{2}=0$ ' in Equation (29), respectively, then we obtain formal solitary wave solutions to Equation (23) as

$$
\begin{aligned}
& u_{n}(t)=a_{0}+\frac{1}{2} \tanh (d \sqrt{-\mu}) \tanh \left(\sqrt{-\mu}\left(d n-\frac{\sinh (2 d \sqrt{-\mu})}{\Gamma(1+\alpha) \sqrt{-\mu}} t^{\alpha}+\chi\right)\right), \\
& u_{n}(t)=a_{0}+\frac{1}{2} \tanh (d \sqrt{-\mu}) \operatorname{coth}\left(\sqrt{-\mu}\left(d n-\frac{\sinh (2 d \sqrt{-\mu})}{\Gamma(1+\alpha) \sqrt{-\mu}} t^{\alpha}+\chi\right)\right),
\end{aligned}
$$

where $a_{0}, d, \chi_{\text {, and }} \mu(<0)$ remain arbitrary.

Similarly, if we let ' $C_{1} \neq 0$ and $C_{2}=0$ ' or ' $C_{1}=0$ and $C_{2} \neq 0$ ' in Equation (32), respectively, then we obtain formal periodic wave solutions to Equation (2) as

$$
\begin{aligned}
& u_{n}(t)=a_{0}-\frac{1}{2} \tan (d \sqrt{\mu}) \tan \left(\sqrt{\mu}\left(d n-\frac{\sin (2 d \sqrt{\mu})}{\Gamma(1+\alpha) \sqrt{\mu}} t^{\alpha}+\chi\right)\right), \\
& u_{n}(t)=a_{0}+\frac{1}{2} \tan (d \sqrt{\mu}) \cot \left(\sqrt{\mu}\left(d n-\frac{\sin (2 d \sqrt{\mu})}{\Gamma(1+\alpha) \sqrt{\mu}} t^{\alpha}+\chi\right)\right),
\end{aligned}
$$

where $a_{0}, d, \chi$, and $\mu(>0)$ remain arbitrary.

\section{Example 4.2}

Consider another time-fractional DDE of rational type:

$$
D_{t}^{\alpha} u_{n}=\frac{4\left(u_{n-1}-u_{n+1}\right) u_{n}^{2}}{\left(1+u_{n-1} u_{n}\right)\left(1+u_{n} u_{n+1}\right)}, 0<\alpha \leq 1
$$

The case $\alpha=1$ of Equation (40), which can be transformed to the discrete modified KdV equation via a real discrete Miura transformation, appears in [41]. Now, we aim to solve Equation (3). In like manner, we first make the fractional complex transformation

$$
u_{n}=U_{n}\left(\xi_{n}\right), \xi_{n}=d n+\frac{k}{\Gamma(1+\alpha)} t^{\alpha}+\chi_{1}
$$

where $d$ and $k$ are real parameters to be specified, whereas $\chi$ denotes the phase shift. Substituting Equation (41) into Equation (40) leads to the equation

$$
k U_{n}^{\prime}\left(1+U_{n-1} U_{n}\right)\left(1+U_{n} U_{n+1}\right)-4\left(U_{n-1}-U_{n+1}\right) U_{n}^{2}=0,
$$

where prime denotes ordinary derivative with respect to the new independent variable $\xi_{n}$. Our procedure suggests then to look for special solutions of Equation (42) in the form

$$
U_{n}=a_{0}+a_{1}\left(\frac{G^{\prime}}{G}\right), a_{1} \neq 0
$$

where $G=G\left(\xi_{n}\right)$ satisfies Equation (13), whereas $a_{0}$ and $a_{1}$ are arbitrary constants to be specified. Because the procedure is similar, from now on, we shall omit some details for the sake of brevity.

4.2.1. Hyperbolic function solutions. In case $\mu<0$, substituting Equation (43) and $U_{n \pm 1}$ along with (13) into Equation (42), clearing the denominator, and setting the coefficients of $\left(\frac{G^{\prime}}{G}\right)^{\prime}(I=0,1, \ldots, 6)$ to zero, we derive a system of nonlinear algebraic equations for $a_{0}$, $a_{1}, d, k$, and $\mu$. Solving the resulting system, we obtain the relations

$$
\begin{aligned}
& \lambda=0, a_{0}=\cosh (d \sqrt{-\mu}), a_{1}= \pm \frac{\sinh (d \sqrt{-\mu})}{\sqrt{-\mu}}, k=-\frac{2 \tanh (d \sqrt{-\mu})}{\sqrt{-\mu}}, \\
& \lambda=0, a_{0}=-\cosh (d \sqrt{-\mu}), a_{1}= \pm \frac{\sin (d \sqrt{-\mu})}{\sqrt{-\mu}}, k=-\frac{2 \tanh (d \sqrt{-\mu})}{\sqrt{-\mu}} .
\end{aligned}
$$


Setting the parameter values (44) and (45) into the expression (43) in accordance with Equation (14), one can construct hyperbolic function solutions to Equation (40) as

$$
\begin{aligned}
& u_{n}(t)=\cosh (d \sqrt{-\mu}) \pm \sinh (d \sqrt{-\mu})\left(\frac{C_{1} \cosh \left(\sqrt{-\mu} \xi_{\mathbf{n}}\right)+C_{2} \sinh \left(\sqrt{-\mu} \xi_{\mathbf{n}}\right)}{C_{1} \sinh \left(\sqrt{-\mu} \xi_{\mathbf{n}}\right)+C_{2} \cosh \left(\sqrt{-\mu} \xi_{\mathbf{n}}\right)}\right), \\
& u_{n}(t)=-\cosh (d \sqrt{-\mu}) \pm \sinh (d \sqrt{-\mu})\left(\frac{C_{1} \cosh \left(\sqrt{-\mu} \xi_{\mathbf{n}}\right)+C_{2} \sinh \left(\sqrt{-\mu} \xi_{\mathbf{n}}\right)}{C_{1} \sinh \left(\sqrt{-\mu} \xi_{\mathbf{n}}\right)+C_{2} \cosh \left(\sqrt{-\mu} \xi_{\mathbf{n}}\right)}\right),
\end{aligned}
$$

where $\xi_{n}=d n-\frac{2 \tanh (d \sqrt{-\mu})}{\Gamma(1+\alpha) \sqrt{-\mu}} t^{\alpha}+\chi$, whereas $d, \chi_{,} \mu(<0), C_{1}$, and $C_{2}$ remain arbitrary.

4.2.2. Trigonometric function solutions. In case $\mu>0$, substituting Equation (43) and $U_{n \pm 1}$ along with (13) into Equation (42), clearing the denominator, and setting the coefficients of $\left(\frac{G^{\prime}}{G}\right)^{l}(I=0,1, \ldots, 6)$ to zero, we derive a system of nonlinear algebraic equations for $a_{0}, a_{1}, d, k$, and $\mu$. Solving the resulting system, we obtain the relations

$$
\begin{aligned}
& \lambda=0, a_{0}=\cos (d \sqrt{\mu}), a_{1}= \pm \frac{\sin (d \sqrt{\mu})}{\sqrt{\mu}}, k=-\frac{2 \tan (d \sqrt{\mu})}{\sqrt{\mu}}, \\
& \lambda=0, a_{0}=-\cos (d \sqrt{\mu}), a_{1}= \pm \frac{\sin (d \sqrt{\mu})}{\sqrt{\mu}}, k=-\frac{2 \tan (d \sqrt{\mu})}{\sqrt{\mu}} .
\end{aligned}
$$

Setting the parameter values (48) and (49) into the expression (43) in accordance with Equation (15), one can construct trigonometric function solutions to Equation (40) as

$$
\begin{aligned}
& u_{n}(t)=\cos (d \sqrt{\mu}) \pm \sin (d \sqrt{\mu})\left(\frac{-C_{1} \sin \left(\sqrt{\mu \xi_{\mathrm{n}}}\right)+C_{2} \cos \left(\sqrt{\mu \xi_{\mathrm{n}}}\right)}{C_{1} \cos \left(\sqrt{\mu \xi_{\mathrm{n}}}\right)+C_{2} \sin \left(\sqrt{\mu \xi_{\mathrm{n}}}\right)}\right) \\
& u_{n}(t)=-\cos (d \sqrt{\mu}) \pm \sin (d \sqrt{\mu})\left(\frac{-C_{1} \sin \left(\sqrt{\mu \xi_{\mathrm{n}}}\right)+C_{2} \cos \left(\sqrt{\mu \xi_{\mathrm{n}}}\right)}{C_{1} \cos \left(\sqrt{\mu \xi_{\mathrm{n}}}\right)+C_{2} \sin \left(\sqrt{\mu \xi_{\mathrm{n}}}\right)}\right)
\end{aligned}
$$

where $\xi_{n}=d n-\frac{2 \tan (d \sqrt{\mu})}{\Gamma(1+\alpha) \sqrt{\mu}} t^{\alpha}+\chi$, whereas $d, \chi, \mu(>0), C_{1}$, and $C_{2}$ remain arbitrary.

4.2.3. Rational function solutions. In case $\mu=0$, substituting Equation (43) and $U_{n \pm 1}$ along with (13) into Equation (42), clearing the denominator, and setting the coefficients of $\left(\frac{G^{\prime}}{G}\right)^{\prime}(I=0,1, \ldots, 5)$ to zero, we derive a system of nonlinear algebraic equations for $a_{0}$, $a_{1}, d$, and $k$. Solving the resulting system, we obtain the relation

$$
\begin{gathered}
\lambda=0, a_{0}=1, a_{1}= \pm d, k=-2 d, \\
\lambda=0, a_{0}=-1, a_{1}= \pm d, k=-2 d .
\end{gathered}
$$

Inserting the parameter values (52) into the expression (43) in accordance with Equation (16), one can construct a rational function solution to Equation (40) as

$$
\begin{aligned}
& u_{n}(t)=1 \pm d\left(\frac{C_{1}}{C_{1}\left(d n-\frac{2 d}{\Gamma(1+\alpha)} t^{\alpha}+\chi\right)+C_{2}}\right) \\
& u_{n}(t)=-1 \pm d\left(\frac{C_{1}}{C_{1}\left(d n-\frac{2 d}{\Gamma(1+\alpha)} t^{\alpha}+\chi\right)+C_{2}}\right)
\end{aligned}
$$

where $d, \chi, C_{1}$, and $C_{2}$ remain arbitrary. 
Example 4.3

Consider the following system of time-fractional DDEs of rational type:

$$
\begin{aligned}
& D_{t}^{\alpha} u_{n}=\frac{\left(v_{n+1}-v_{n-1}\right)\left(1-u_{n}^{2}\right)\left(1-v_{n}^{2}\right)}{\left(v_{n-1}+v_{n}\right)\left(v_{n}+v_{n+1}\right)}, 0<\alpha \leq 1, \\
& D_{t}^{\alpha} v_{n}=\frac{\left(u_{n+1}-u_{n-1}\right)\left(1-u_{n}^{2}\right)\left(1-v_{n}^{2}\right)}{\left(u_{n-1}+u_{n}\right)\left(u_{n}+u_{n+1}\right)}, 0<\alpha \leq 1 .
\end{aligned}
$$

The case $\alpha=1$ of the system (56), which is related to the self-dual network equations via a real discrete Miura transformation, appears in [42]. Like before, we first make the fractional complex transformation

$$
u_{n}=U_{n}\left(\xi_{n}\right), v_{n}=V_{n}\left(\xi_{n}\right), \xi_{n}=d n+\frac{k}{\Gamma(1+)} t^{\alpha}+\chi_{1}
$$

where $d$ and $k$ are real parameters to be specified, whereas $\chi$ denotes the phase shift. Substituting Equation (57) into Equation (56) leads to the system

$$
\begin{aligned}
& k U_{n}^{\prime}\left(V_{n-1}+V_{n}\right)\left(V_{n}+V_{n+1}\right)-\left(V_{n+1}-V_{n-1}\right)\left(1-U_{n}^{2}\right)\left(1-V_{n}^{2}\right)=0 \\
& k V_{n}^{\prime}\left(U_{n-1}+U_{n}\right)\left(U_{n}+U_{n+1}\right)-\left(U_{n+1}-U_{n-1}\right)\left(1-U_{n}^{2}\right)\left(1-V_{n}^{2}\right)=0,
\end{aligned}
$$

where prime denotes derivative with respect to the new independent variable $\xi_{n}$. Our procedure suggests then to look for special solutions of the system (31) in the form

$$
U_{n}=a_{0}+a_{1}\left(\frac{G^{\prime}}{G}\right), a_{1} \neq 0 ; v_{n}=b_{0}+b_{1}\left(\frac{G^{\prime}}{G}\right), b_{1} \neq 0,
$$

where $G=G\left(\xi_{n}\right)$ satisfies Equation (13), whereas $a_{0}, a_{1}, b_{0}$, and $b_{1}$ are arbitrary constants to be specified. Because the procedure is very similar, we just present the results.

\subsubsection{Hyperbolic function solutions.}

$$
\begin{aligned}
& u_{n}(t)= \pm \frac{\sqrt{1-2 b_{1} \sqrt{-\mu} \operatorname{coth}(d \sqrt{-\mu})-\mu b_{1}^{2}}}{1-2 b_{1} \sqrt{-\mu} \operatorname{coth}(d \sqrt{-\mu})}+\frac{b_{1} \sqrt{-\mu}}{1-2 b_{1} \sqrt{-\mu} \operatorname{coth}(d \sqrt{-\mu})}\left(\frac{C_{1} \cosh \left(\sqrt{-\mu} \xi_{\mathbf{n}}\right)+C_{2} \sinh \left(\sqrt{-\mu} \xi_{\mathbf{n}}\right)}{C_{1} \sinh \left(\sqrt{-\mu} \xi_{\mathbf{n}}\right)+C_{2} \cosh \left(\sqrt{-\mu} \xi_{\mathbf{n}}\right)}\right) \\
& v_{n}(t)= \pm \sqrt{1-2 b_{1}} \sqrt{-\mu} \operatorname{coth}(d \sqrt{-\mu}) b_{1}-\mu b_{1}^{2}+b_{1} \sqrt{-\mu}\left(\frac{C_{1} \cosh \left(\sqrt{-\mu} \xi_{\mathbf{n}}\right)+C_{2} \sinh \left(\sqrt{-\mu} \xi_{\mathbf{n}}\right)}{C_{1} \sinh \left(\sqrt{-\mu} \xi_{\mathbf{n}}\right)+C_{2} \cosh \left(\sqrt{-\mu} \xi_{\mathbf{n}}\right)}\right)
\end{aligned}
$$

where $\xi_{n}=d n-\frac{2 b_{1}^{2} \sqrt{-\mu}}{\Gamma(1+\alpha)\left(\tanh (d \sqrt{-\mu})-2 b_{1} \sqrt{-\mu}\right)} t^{\alpha}+\chi$, whereas $b_{1}, d, \chi, \mu(<0), C_{1}$, and $C_{2}$ remain arbitrary.

\subsubsection{Trigonometric function solutions.}

$$
\begin{aligned}
& u_{n}(t)=\mp \frac{\sqrt{1-2 b_{1} \sqrt{\mu} \cot (d \sqrt{\mu})-\mu b_{1}^{2}}}{\left(1-2 b_{1} \sqrt{\mu} \cot (d \sqrt{\mu})\right)}-\frac{b_{1} \sqrt{\mu}}{\left(1-2 b_{1} \sqrt{\mu} \cot (d \sqrt{\mu})\right)}\left(\frac{-C_{1} \sin \left(\sqrt{\mu} \xi_{\mathrm{n}}\right)+C_{2} \cos \left(\sqrt{\mu} \xi_{\mathrm{n}}\right)}{C_{1} \cos \left(\sqrt{\mu} \xi_{\mathrm{n}}\right)+C_{2} \sin \left(\sqrt{\mu} \xi_{\mathrm{n}}\right)}\right), \\
& v_{n}(t)= \pm \sqrt{1-2 b_{1} \sqrt{\mu} \cot (d \sqrt{\mu})-\mu b_{1}^{2}}+b_{1} \sqrt{\mu}\left(\frac{-C_{1} \sin \left(\sqrt{\mu} \xi_{\mathrm{n}}\right)+C_{2} \cos \left(\sqrt{\mu} \xi_{\mathrm{n}}\right)}{C_{1} \cos \left(\sqrt{\mu} \xi_{\mathrm{n}}\right)+C_{2} \sin \left(\sqrt{\mu} \xi_{\mathrm{n}}\right)}\right),
\end{aligned}
$$

where $\xi_{n}=d n+\frac{2 b_{1}^{2} \sqrt{\mu}}{\Gamma(1+\alpha)\left(\tan (d \sqrt{\mu})-2 b_{1} \sqrt{\mu}\right)} t^{\alpha}+\chi$, whereas $b_{1}, d, \chi_{1} \mu(>0), C_{1}$, and $C_{2}$ remain arbitrary.

\subsubsection{Rational function solutions.}

$$
\begin{aligned}
& u_{n}(t)=\mp \frac{d}{d-2 b_{1}} \sqrt{1-\frac{2 b_{1}}{d}}-\frac{d b_{1}}{d-2 b_{1}}\left(\frac{C_{1}}{C_{1}\left(d n+\frac{2 b_{1}^{2}}{\Gamma(1+\alpha)\left(d-2 b_{1}\right)} t^{\alpha}+\chi\right)+C_{2}}\right) \\
& v_{n}(t)= \pm \sqrt{1-\frac{2 b_{1}}{d}}+b_{1}\left(\frac{C_{1}}{C_{1}\left(d n+\frac{2 b_{1}^{2}}{\Gamma(1+\alpha)\left(d-2 b_{1}\right)} t^{\alpha}+\chi\right)+C_{2}}\right)
\end{aligned}
$$

where $b_{1}, d, \chi_{,} C_{1}$, and $C_{2}$ remain arbitrary. 
Remark 3

As in Example 4.1, if desired, one can assign special values to the involved arbitrary parameters in the results of the Examples 4.2 and 4.3 for further analysis. This procedure is also skipped for the sake of brevity.

\section{Conclusion}

Recent advances of fractional calculus are stimulated by new examples of applications in science and engineering. In this paper, a generalization of DDEs of rational type is made by means of Jumarie's modified Riemann-Liouville derivative. These models are important from theoretical and applied point of view. Novel results are being derived for the considered equations indicating that the field of fractional calculus is very reach and interesting. As a result, a successful attempt on modeling time-fractional DDEs of rational type has been made via an improved version of the (G'/G)-expansion method. Of course, symbolic computation systems (such as MATHEMATICA, MATLAB, and MAPLE) played a crucial role in the computations. Our examples convinced us that the used fractional complex transformations are extremely helpful for converting FDEs with Jumarie's sense to ODEs. Such transformations correspond to only wave solutions. It should not be surprising that some classes of DDEs of fractional order may not be suited for our method. For example, one cannot obtain real-valued solutions for the equation

$$
D_{t}^{\alpha} u_{n}=\frac{\left(u_{n-1}-u_{n+1}\right)\left(1-u_{n}^{2}\right)\left(\eta^{2}-\mu^{2} u_{n}^{2}\right)}{\left(u_{n-1}+u_{n}\right)\left(u_{n}+u_{n+1}\right)}, 0<\alpha \leq 1,
$$

where $\eta$ and $\mu$ are taken as arbitrary parameters. This remains an open research problem. Indeed, the case $\alpha=1$ of Equation (64) was considered in [42]. In addition, it is worth to mention here that we could not give further details about the real physical meaning of our exact solutions because of the lack of theoretical and experimental basis related to them. We believe that our findings will bring some new insights into the field of fractional calculus and will be used in applications. For future work, our next step will be the analytic investigation of DDEs with fractional order using some recent approaches such as Exp-function method [43-46].

\section{Acknowledgements}

The author is grateful to the anonymous referee(s) for the valuable suggestions.

\section{References}

1. Miller KS, Ross B. An Introduction to the Fractional Calculus and Fractional Differential Equations. Wiley: New York, 1993.

2. Oldham KB, Spanier J. The Fractional Calculus. Academic Press: New York, London, 1974.

3. Podlubny I. Fractional Differential Equations. Academic Press: San Diego, 1999.

4. Samko SG, Kilbas AA, Marichev OI. Fractional Integrals and Derivatives, Theory and Applications. Gordon and Breach: Yverdon, 1993.

5. Kilbas AA, Srivastava HM, Trujillo JJ. Theory and Applications of Fractional Differential Equations. Elsevier: San Diego, 2006.

6. Jumarie G. Modified Riemann-Liouville derivative and fractional Taylor series of nondifferentiable functions further results. Computers \& Mathematics with Applications 2006; 51:1367-1376.

7. Cresson J. Non-differentiable variational principles. Journal of Mathematical Analysis and Applications 2005; 307:48-64.

8. Kolwankar KM, Gangal AD. Local fractional Fokker-Planck equation. Physical Review Letters 1998; 80:214-217.

9. Cui M. Compact finite difference method for the fractional diffusion equation. Journal of Computational Physics 2009; 228:7792-7804.

10. Huang Q, Huang G, Zhan H. A finite element solution for the fractional advection-dispersion equation. Advances in Water Resources $1578 ; 31$.

11. Odibat Z, Momani S. A generalized differential transform method for linear partial differential equations of fractional order. Applied Mathematics Letters 2008; 21:194-199.

12. El-Sayed AMA, Gaber M. The Adomian decomposition method for solving partial differential equations of fractal order in finite domains. Physics Letters A 2006; 359:175-182.

13. Odibat $Z$, Momani $S$. The variational iteration method: An efficient scheme for handling fractional partial differential equations in fluid mechanics. Computers \& Mathematics with Applications 2009; 58:2199-2208.

14. He JH. A coupling method of a homotopy technique and a perturbation technique for non-linear problems. International Journal of Non-Linear Mechanics 2000; 35:37-43.

15. Lu B. The first integral method for some time fractional differential equations. Journal of Mathematical Analysis and Applications 2012; 395:684-693.

16. Zhang S, Zhang HQ. Fractional sub-equation method and its applications to nonlinear fractional PDEs. Physics Letters A 2011; 375:1069-1073.

17. Lakestani M, Dehghan M, Irandoust-pakchin S. The construction of operational matrix of fractional derivatives using B-spline functions. Communications in Nonlinear Science and Numerical Simulation 2012; 17:1149-1162.

18. Saadatmandi A, Dehghan M. A tau approach for solution of the space fractional diffusion equation. Computers \& Mathematics with Applications 2011 ; 62:1135-1142.

19. Dehghan M, Manafian J, Saadatmandi A. Solving nonlinear fractional partial differential equations using the homotopy analysis method. Numerical Methods for Partial Differential Equations 2010; 26:448-479.

20. Dehghan M, Manafian J, Saadatmandi A. The solution of the linear fractional partial differential equations using the homotopy analysis method. Zeitschrift fur Naturforschung 2010; 65a:935-949.

21. Saadatmandi A, Dehghan M. A new operational matrix for solving fractional-order differential equations. Computers \& Mathematics with Applications 2010; 59:1326-1336.

22. Fermi E, Pasta J, Ulam S. Collected Papers of Enrico Fermi. University of Chicago Press: Chicago, 1965.

23. Toda M. Theory of Nonlinear Lattices. Springer-Verlag: New-York, 1989. 
24. Dai $C Q$, Wang YY. Exact traveling wave solutions of the discrete nonlinear Schrödinger equation and the hybrid lattice equation obtained via the exp-function method. Physica Scripta 2008; 78:015013.

25. Ma WX, You Y. Rational solutions of the Toda lattice equation in Casoratian form. Chaos, Solitons \& Fractals 2004; 22:395-406.

26. Zhu SD, Chu YM, Qiu SL. The homotopy perturbation method for discontinued problems arising in nanotechnology. Computers \& Mathematics with Applications 2009; 58:2398-2401.

27. Yang P, Chen Y, Li ZB. ADM-Padé technique for the nonlinear lattice equations. Applied Mathematics and Computation 2009; 210:362-375.

28. Hu XB, Ma WX. Application of Hirota's bilinear formalism to the Toeplitz lattice-some special soliton-like solutions. Physics Letters A 2002; 293:161-165.

29. Zhang S, Dong L, Ba JM, Sun YN. The (G'/G)-expansion method for nonlinear differential-difference equations. Physics Letters A 2009; 373:905-910.

30. Saadatmandi A, Dehghan M. Numerical solution of the higher-order linear Fredholm integro-differential-difference equation with variable coefficients. Computers \& Mathematics with Applications 2010; 59:2996-3004.

31. He JH, Wu GC, Austin F. The variational iteration method which should be followed. Nonlinear Science Letters A 2010; 1:1-30.

32. Zhang S, Dong L, Ba JM, Sun YN. The (G'/G)-expansion method for a discrete nonlinear Schrödinger equation. Pramana - Journal of Physics 2010; 74:207-218.

33. Bin Z. (G'/G)-expansion method for solving fractional partial differential equations in the theory of mathematical physics. Communications in Theoretical Physics 2012; 58:623-630.

34. Gepreel KA, Omran S. Exact solutions for nonlinear partial fractional differential equations. Chinese Physics B 2012; 21:110204.

35. Jumarie G. Laplace's transform of fractional order via the Mittag-Leffler function and modified Riemann-Liouville derivative. Applied Mathematics Letters 2009; 22:1659-1664.

36. Wu GC, Lee EWM. Fractional variational iteration method and its application. Physics Letters A 2010; 374:2506-2509.

37. Jumarie G. New stochastic fractional models for Malthusian growth, the Poissonian birth process and optimal management of populations. Mathematical and Computer Modelling 2006; 44:231-254.

38. Li ZB, He JH. Fractional complex transformation for fractional differential equations. Computers \& Mathematics with Applications 2010; 15:970-973.

39. Aslan I. A note on the (G'/G)-expansion method again. Applied Mathematics and Computation 2010; 217:937-938.

40. Narita K. New discrete modified KdV equation. Progress of Theoretical Physics 1991; 86:817-824.

41. Narita K. N-soliton solution of a lattice equation related to the discrete MKdV equation. Journal of Mathematical Analysis and Applications 2011; 381:963-965.

42. Narita K. Solutions for a system of difference-differential equations related to the self-dual network equation. Progress of Theoretical Physics 2001; 106:1079-1096.

43. Dehghan M, Manafian J, Saadatmandi A. Analytical treatment of some partial differential equations arising in mathematical physics by using the exp-function method. International Journal of Modern Physics B 2011; 25:2965-2981.

44. Dehghan M, Heris JM. Application of the exp-function method for solving a partial differential equation arising in biology and population genetics. International Journal for Numerical Methods in Heat and Fluid Flow 2011; 21:736-753.

45. Aslan I, Marinakis V. Some remarks on Exp-function method and its applications. Communications in Theoretical Physics 2011; 56:397-403.

46. Aslan I. Some remarks on Exp-function method and its applications-A supplement. Communications in Theoretical Physics 2013; 60:521-525. 